\title{
Ulnar Nerve Morphology in Magnetic Resonance Imaging Predicts Nerve Recovery After Surgery for Cubital Tunnel Syndrome
}

\section{Jong Seop Kim}

Seoul National University Bundang Hospital, Seoul National University College of Medicine

\section{Young Ju Chae}

Seoul National University Bundang Hospital, Seoul National University College of Medicine

\section{Gajendra Mani Shah}

Department of orthopedics and trauma surgery, patan academy of health sciences, Lalipur, Nepar

Hyun Sik Gong ( $\sim$ hsgong@snu.ac.kr)

Seoul National University Bundang Hospital, Seoul National University College of Medicine https://orcid.org/0000-0003-4028-1559

\section{Research article}

Keywords: Magnetic resonance imaging, electrodiagnostic study, ulnar nerve, morphology, cubital tunnel syndrome, recovery, surgery

Posted Date: October 22nd, 2020

DOl: https://doi.org/10.21203/rs.3.rs-90885/v1

License: (1) This work is licensed under a Creative Commons Attribution 4.0 International License. Read Full License

Version of Record: A version of this preprint was published at Hand Surgery and Rehabilitation on November 1st, 2021. See the published version at https://doi.org/10.1016/j.hansur.2021.10.316. 


\section{Abstract}

Background: Magnetic resonance imaging (MRI) is helpful for the diagnosis of cubital tunnel syndrome (CuTS), but its prognostic value for surgical outcomes is unknown. We aimed to determine whether MRI parameters correlated with outcomes after surgery for CuTS.

Methods: We reviewed 40 patients who had electrodiagnostic tests and MRIs for CuTS preoperatively and had 6-month evaluations postoperatively. The MRI parameters were ulnar nerve cross-sectional area (UNCSA) measured at 6 different levels around the medial epicondyle (ME), signal intensity changes of innervated muscles of the ulnar nerve, and the presence of ganglion around the ulnar nerve. Other factors assessed were age, symptom duration, symptom severity, presence of diabetes mellitus, and electrodiagnostic parameters including motor nerve conduction velocity $(\mathrm{mNCV})$. We analyzed the factors associated with fair or poor outcomes graded by Wilson-Krout classification.

Results: The UNCSA was the largest at ME level and smallest at $3 \mathrm{~cm}$ distal to ME level. Increased ulnar nerve signal intensity changes were found in 34 subjects and increased forearm muscle signal intensity changes were found in two. Ten patients were found to have ganglia.

Twelve patients (30\%) had excellent results, $19(48 \%)$ had good, $8(20 \%)$ had fair, and $1(4 \%)$ had a poor result. In univariate analysis, fair or poor outcomes were associated with increased UNCSA $1 \mathrm{~cm}$ proximal and $1 \mathrm{~cm}$ distal from the $\mathrm{ME}$, the presence of ganglion, and decreased $\mathrm{mNCV}$. In multivariate analysis, fair or poor outcomes were associated with either increased UNCSA $1 \mathrm{~cm}$ distal from the ME (OR 11.15; $p=0.019)$, or increased UNCSA $1 \mathrm{~cm}$ proximal from the ME (OR 16.01; $p=0.038)$ and decreased $\mathrm{mNCV}(\mathrm{OR} 0.92 ; \mathrm{p}=0.044)$.

Conclusions: This study demonstrated that increased ulnar nerve cross-sectional area on MRI correlated with suboptimal improvement after surgery for CuTS at 6 months follow up. MRI examination for morphologic changes of the ulnar nerve can be helpful for patient consultation on the prognosis of surgery for CuTS.

\section{Background}

Cubital tunnel syndrome (CUTS) is the second most common nerve entrapment syndrome affecting the upper extremity.[1,2] The diagnosis of CuTS is based on clinical signs and symptoms, which include both motor and sensory abnormalities involving the ulnar nerve distribution over the forearm and hand. Electrodiagnostic test (EDX) and magnetic resonance imaging (MRI) can help confirm a diagnosis, quantifying the severity of the lesion, and identifying the exact site of the ulnar nerve compression. $[3,4]$

Several prognostic factors for surgical outcomes have been evaluated for CuTS, including age, duration of symptoms, stage of the disease, smoking, diabetes mellitus (DM), and ulnar nerve cross-sectional area (CSA) measured by ultrasonography.[5-8] Factors that have been shown to have poor outcomes are 
intraoperative intraneural fibrosis, preoperative intrinsic muscle weakness, prolonged duration of symptoms, and advanced age.[8]

While EDX can evaluate functional aspects of the ulnar nerve, MRI can show its morphological changes including the nerve size and signal changes independent of the proficiency of the examiner.[9] Although previous studies using MRI have shown its diagnostic value, studies are lacking on the value of MRI for surgical outcomes.[10,11] Thus, the purpose of this study was to determine whether ulnar nerve morphology in MRI correlated with outcomes after surgery for CuTS.

\section{Methods}

\section{Patients}

We obtained approval from the Institutional Review Board of our institution for this study. We reviewed the medical records of patients who had surgery for CuTS at our hospital, a referral, training hospital located in an urban area, between January 2014 and June 2019. The inclusion criteria were a diagnosis of CUTS and the presence of both EDX and MRI examinations.

Initially, we identified 107 patients who underwent surgery for CuTS and included 78 patients who had both EDX and MRI examinations. Some of the EDX-positive patients did not have MRI examinations due to reasons such as claustrophobia, cardiac pacemakers, and inadequate insurance coverage, and thus were not included in the study. The exclusion criteria were revision surgery, the presence of other compressive neuropathies or cervical radiculopathy, neuromuscular disease, and lack of follow-up examination at 6 months. From this 78, we excluded 38 patients according to the exclusion criteria and finally analyzed 40 patients (Fig. 1). There were 27 men and 13 women in the study with the mean ( \pm SD) age of $48( \pm 17)$ years (range, 17-75 years).

The patients underwent ulnar nerve decompression at the elbow and an epicondylectomy under general anesthesia, followed by one week of immobilization and no formal physical therapy thereafter. All operations were performed by a single surgeon.

\section{MRI evaluation}

All patients were imaged in the supine position with the examined arm in an adducted and stretched position, using a 3-Tesla system MR unit (Ingenia or Ingenia Cx, Philips Healthcare, the Netherlands) with a dedicated elbow-coil. The MRI parameters evaluated were 1) ulnar nerve CSA, 2) nerve and muscle signal intensity changes, and 3 ) the presence or absence of ganglion.

The ulnar nerve CSA was measured on axial T2-weighted fast spin-echo sequences (TR/TE, 2,900$3,200 / 75-100$, section thickness $2.5 \mathrm{~mm}$, intersection gap $0.25 \mathrm{~mm}$, number of Sect. 40, field of view $100 \times 100 \mathrm{~mm}$, and acquisition matrix $256 \times 256$ ). The CSA was measured at 6 points; $2 \mathrm{~cm}$ and $1 \mathrm{~cm}$ proximal from the medial epicondyle (ME), at the $M E$, and $1 \mathrm{~cm}, 2 \mathrm{~cm}$, and $3 \mathrm{~cm}$ distal to the ME. We 
measured the longer diameter (a) and shorter diameter (b) of the ulnar nerve and calculated the CSA by using formula Area $=\pi \times a / 2 X b / 2$ (Fig. 2A).

Signal intensity changes of the ulnar nerve (Fig. 2B) and hand and forearm flexor muscles (Fig. 2C) were assessed on T2-weighted images in axial section views. We examined any space occupying lesion around the ulnar nerve including medial osteophyte, medial side joint fluid collection, and ganglia (Fig. 2D).

One radiologist specializing in musculoskeletal imaging recorded the signal intensity changes of the nerve and muscles and two orthopedic surgeons measured the CSA. We tested intra-rater reliability of the CSA measurement by repeating all measurements after two weeks and inter-rater reliability of the independent assessments by two examiners. There was no significant difference between the two examiners. The intraclass correlation coefficient of the intra-rater reliability and that of the inter-rater reliability were good-to-excellent (Table 1).[10] Therefore, we used the CSA measured by one of the raters.

\section{Table 1. Intraclass coefficient (ICC) of inter \& intra-rater reliabilities}

$\begin{array}{lll} & \text { Inter-rater reliability }(95 \% \mathrm{Cl}) & \text { Intra-rater reliability }(95 \% \mathrm{Cl}) \\ \text { CSA-3 } & 0.98(0.95,0.99) & 0.96(0.92,0.99) \\ \text { CSA-2 } & 0.92(0.87,0.97) & 0.91(0.81,0.96) \\ \text { CSA-1 } & 0.96(0.91,0.98) & 0.92(0.83,0.97) \\ \text { CSAO } & 0.98(0.95,0.99) & 0.95(0.90,0.98) \\ \text { CSA1 } & 0.92(0.83,0.97) & 0.85(0.68,0.94) \\ \text { CSA2 } & 0.95(0.88,0.98) & 0.92(0.83,0.97)\end{array}$

$C S A=$ Cross sectional area of the ulnar nerve; $C S A-3=C S A$ at $3 \mathrm{~cm}$ distal to the medial epicondyle; $C S A O=C S A$ at the medial epicondyle; $C S A 2=C S A$ at 2 proximal to the medial epicondyle.

\section{EDX evaluation}

All EDXs were performed following the guidelines of the American Association of Electrodiagnostic Medicine at the EDX study laboratory in our institute.[12] The confirmatory criteria included: (1) motor nerve conduction velocity ( $\mathrm{mNCV}$ ) across the elbow of less than $50 \mathrm{~m} / \mathrm{s}$, (2) an $\mathrm{mNCV}$ difference of greater than $10 \mathrm{~m} / \mathrm{s}$ between the elbow segment and the forearm segment, and (3) a decrease of the compound muscle action potential (CMAP) amplitude from below the elbow to above the elbow greater than $20 \%$, suggesting a conduction block.

\section{Clinical evaluation}

Preoperatively, we evaluated the severity of the symptoms which was classified according to McGowan grades.[13] Patients with subjective symptoms and no abnormal objective findings were classified as 
grade I, patients with good intrinsic strength and no detectable muscle atrophy as grade lla, patients with fair intrinsic strength and detectable muscle atrophy as grade $\mathrm{Ilb}$, and patients with profound sensory and motor disturbances with marked intrinsic atrophy as grade III. Other clinical parameters evaluated were the age at surgery, gender, body mass index (BMI), the presence of diabetes mellitus (DM), and duration of the symptoms.

At 6-months postoperatively, clinical evaluation of nerve recovery was conducted using the modified Wilson-Krout criteria.[14] Patients with complete return of sensation and function to the hand without discomfort were graded as excellent; return of a functional hand without discomfort, but with residual weakness or diminished sensation were graded as good; and improvement in only one area, comfort, strength, or sensation, were graded as fair; and those with no improvement or a worsened condition were graded as poor.

\section{Statistical Analysis}

We evaluated the surgical outcomes by logistic regression analyses. The dependent variable was the postoperative Wilson-Krout grades and the independent variables were the MRI parameters (ulnar nerve CSA at 6 levels, signal intensity changes of the ulnar nerve and forearm muscles, and presence of space occupying lesion), EDX parameters (mNCV and CMAP amplitude), and clinical parameters (age at surgery, $B M I$, presence of DM, symptom duration, and preoperative symptom severity as assessed by McGowan grade). We performed univariate and multivariate logistic regression analyses for the variables associated with fair or poor Wilson-Krout grades. Variables with p-values less than 0.1 in univariate analyses were selected for multivariate analysis. We also calculated the area under the curve (AUC) for the ulnar nerve CSA to determine a cutoff value for fair or poor outcomes with maximum sensitivity and specificity.

$P$-values less than 0.05 were considered significant. This analysis was performed using open-source statistical software $R$, version 3.5.1.

\section{Results}

\section{MRI findings}

The ulnar nerve CSA was the largest at ME level and smallest at $3 \mathrm{~cm}$ distal to ME level (Fig. 3). Increased ulnar nerve signal intensity changes were found in 34 subjects and increased forearm muscle signal intensity changes were found in two. Ten patients were found to have space occupying lesions around the ulnar nerve (Table 2). 
Table 2

Baseline characteristics and surgical outcome

\begin{tabular}{|ll|}
\hline Characteristics & Value or number \\
\hline Age (years) & $48 \pm 17$ \\
\hline Sex (Male : Female) & $27: 13$ \\
\hline Body mass index $\left(\mathrm{kg} / \mathrm{m}^{2}\right)$ & $26.4 \pm 4.0$ \\
\hline Right / Left & $22 / 18$ \\
\hline Diabetes mellitus & 8 \\
\hline Symptom severity (McGowan grade) & $0(0 \%)$ \\
Grade I & $15(38 \%)$ \\
Grade Ila & $19(48 \%)$ \\
Grade IIb & $6(15 \%)$ \\
Grade III & \\
\hline Motor nerve conduction velocity (m/s) & $35.1 \pm 13.1$ \\
\hline Compound muscle action potential amplitude (m/V) & $6.2 \pm 5.2$ \\
\hline Surgical outcome (Wilson-Krouts criteria) & $12(30 \%)$ \\
Excellent & $19(48 \%)$ \\
Good & $8(20 \%)$ \\
Fair & $1(4 \%)$ \\
\hline Poor & \\
\hline Values are expressed as the mean \pm standard deviation or number (percentage). & \\
\hline COrS Of nerVe reCOVery & \\
\hline
\end{tabular}

Preoperatively, 15 patients were classified as grade IIA, 19 grade IIB, and six grade III by McGowan grade. At 6-months postoperatively, 12 patients (30\%) had excellent results, 19 (48\%) had good, eight (20\%) had fair and one (4\%) had a poor result according to the Wilson-Krout criteria (Table 3). 
Table 3

\section{Value or number}

Ulnar nerve cross sectional area $\left(C S A, \mathrm{~mm}^{2}\right)$

CSA2

$14.1 \pm 3.9$

CSA1

$17.8 \pm 5.7$

CSAO

$19.8 \pm 6.7$

CSA-1

$17.5 \pm 7.5$

CSA-2

$14.0 \pm 6.4$

CSA-3

$12.6 \pm 6.6$

Signal change

Ulnar nerve

Forearm muscle

Space occupying lesion

10

$C S A 2=C S A$ at $2 \mathrm{~cm}$ proximal to the medial epicondyle; $C S A O=C S A$ at the medial epicondyle; $C S A-3=$ CSA at $3 \mathrm{~cm}$ distal to the medial epicondyle

In univariate analyses, fair or poor outcomes were significantly associated with increased ulnar nerve CSA $1 \mathrm{~cm}$ proximal (OR 11.08 [95\% Cl 1.73 to 218.86]; $p=0.032$ ) and $1 \mathrm{~cm}$ distal from the epicondyle (OR 8.33 [95\% Cl 1.71 to 49.86]; $p=0.012$ ), the presence of space occupying lesion (OR 6.50 [95\% Cl 1.31 to 36.07 ]; $p=0.024)$, and decreased mNCV (OR 0.93 [95\% Cl 0.85 to 0.99]; $p=0.035$ ). No association was found for factors such as age at surgery, BMI, the presence of DM, symptom duration, symptom severity (McGowan grade), ulnar nerve CSA at other sites, signal intensity changes of the ulnar nerve and forearm muscles, and CMAP amplitude (Additional file 1).

In the multivariate analysis, we analyzed each CSA separately because ulnar nerve CSAs measured in the same patient have collinearity. In the multivariate analysis model 1 including ulnar nerve CSA $1 \mathrm{~cm}$ distal from the epicondyle, fair or poor outcomes were associated with increased ulnar nerve CSA $1 \mathrm{~cm}$ distal from the epicondyle (OR 11.15 [95\% Cl 1.74 to 112.75]; $p=0.019)$ only. In multivariate analysis model 2 including ulnar nerve CSA $1 \mathrm{~cm}$ proximal from the epicondyle, fair or poor outcomes were associated with both increased ulnar nerve CSA $1 \mathrm{~cm}$ proximal from the epicondyle (OR 16.01 [95\% Cl 1.76 to 461.14]; $p=$ 0.038) and decreased mNCV (OR 0.92 [95\% Cl 0.83 to 0.99]; $p=0.044)$.

The AUC was calculated for the ulnar nerve CSA to detect fair or poor outcomes. The cut-off value of CSA at distal $1 \mathrm{~cm}$ was $18.9 \mathrm{~mm}^{2}$, whereas the cut-off value of CSA at proximal $1 \mathrm{~cm}$ was $16.7 \mathrm{~mm}^{2}$. Sensitivity and specificity at distal $1 \mathrm{~cm}$ were $80.6 \%$ and $66.7 \%$, and those at proximal $1 \mathrm{~cm}$ were $92.9 \%$ and $50.0 \%$, 
respectively. The AUC for CSA at distal $1 \mathrm{~cm}$ was 0.729 and the AUC for CSA at proximal $1 \mathrm{~cm}$ was 0.724 (Fig. 4).

\section{Discussion}

MRI can evaluate morphology of the ulnar nerve in patients with CUTS while EDX evaluates functional aspects of the nerve. This study demonstrated that fair or poor outcomes after surgery was strongly associated with increased ulnar nerve CSA in MRI (OR $=11.15$ at $1 \mathrm{~cm}$ distal from the ME and OR= 16.01 at $1 \mathrm{~cm}$ proximal from the $M E)$, while the association was minimal with preoperative $E D X(O R=$ 0.92) and no association was seen with preoperative symptom severity as McGowan grades. This result suggests that morphologic changes of the ulnar nerve can better predict delayed nerve recovery after surgery for CUTS than preoperative symptom severity or EDX examination.

In our study, ulnar nerve CSA was increased $1 \mathrm{~cm}$ proximal to the $M E$, at the level of the $M E$, and at $1 \mathrm{~cm}$ distal to the ME, which is consistent with previous studies of MR neurography or ultrasonography.[3, 1517] An MRI study by Bäumer et al reported that the mean ( $\pm S D$ ) ulnar nerve CSA across the cubital tunnel was $15.4 \pm 0.9 \mathrm{~mm}^{2}$, whereas the mean $\left( \pm S D\right.$ ) of healthy control subjects was $11.0 \pm 0.7 \mathrm{~mm}^{2}$. They also suggested that nerve caliber enlargement discriminated severe from mild disease preoperatively and that the mean $( \pm S D)$ ulnar nerve CSA was significantly higher in patients with severe disease $\left(19.4 \pm 2.5 \mathrm{~mm}^{2}\right)$ than the mean $( \pm S D)$ ulnar nerve CSA in patients with mild disease $\left(12.7 \pm 1.2 \mathrm{~mm}^{2}\right) .[15]$

Previous animal model studies have suggested that in compressive neuropathy, peripheral nerves compressed at a local level interfere with both anterograde and retrograde axonal transport and undergo perineurial thickening, fibrosis, and swelling proximal and distal to the compressed segment.[18, 19] Studies have also postulated that increases in nerve caliber are associated with extrinsic injury in which peripheral nerves undergo degenerative demyelination and compensatory remyelination, increasing myelin sheath thickness.[20,21] In CUTS, these pathological alterations occur along the ulnar nerve proximal and distal to the cubital tunnel, and thus increase the ulnar nerve CSA.[17] In this study, fair or poor outcomes were associated with increased ulnar nerve CSA, which reflected morphological changes in the nerve and the severity of the disease. This association has been reported in previous studies using ultrasound.[22, 23] Beekman et al.[22] showed that significant nerve enlargement found during sonography at the time of diagnosis was associated with a poor outcome with an odds ratio of 2.9. A similar study by Domenico et al.[23] also showed that increased ulnar nerve CSAs in preoperative ultrasounds were associated with poor outcomes.

We found that decreased $m N C V$ correlated with fair or poor surgical outcomes in univariate analysis. However, this correlation disappeared in the multivariate model 1 and the OR was only 0.92 in the multivariate model 2. Previous studies have reported on the relationship between preoperative EDX and CSA or between EDX and grip/pinch strength.[16, 19] The relationship between preoperative EDX and postoperative outcomes has been contradictory. A systematic review of the predictors of surgical outcomes after anterior transposition of the ulnar nerve for CUTS found that out of nine articles available, 
four case series and one cohort study reported no association between preoperative EDX and outcomes, whereas one randomized controlled trial, two cases series, and one cohort study reported a significant association.[24]

There were several limitations to this study. First, this study involved patients who underwent surgery, and thus the MRI findings may not apply to the general population of CUTS patients, including those with early symptoms. In addition, many patients had to be excluded due to the exclusion criteria, which may limit the applicability of the study findings. Second, the 6-month follow up period is short to evaluate the final surgical outcomes. Hironori et al.[25] reported that symptoms had improved in 14 out of 15 patients within 4.5 years and nerve conduction velocity had recovered two years after surgery. However, the 6month outcome can be helpful for patient consultation regarding their expectations about recovery speed.

\section{Conclusions}

This study demonstrated that increased ulnar nerve cross-sectional area on MRI correlated with suboptimal improvement after surgery for CUTS at 6 months follow up. This study also suggests that morphologic changes of the ulnar nerve can better predict delayed nerve recovery than preoperative symptom severity or EDX examination. Assessment of morphologic changes of the ulnar nerve can be helpful for patient consultation on the prognosis of surgery for CUTS.

\section{Abbreviations}

$M R I$

Magnetic resonance imaging; CUTS:Cubital tunnel syndrome; EDX:Electrodiagnostic test; UNCSA:UInar nerve cross-sectional area; ME:Medial epicondyle; $m N C V$ :Motor nerve conduction velocity; OR:Odds ratio; DM:Diabetes mellitus; CSA:Cross-sectional area; SD:Standard deviation; CMAP:Compound muscle action potential; BMI:Body mass index; AUC:Area under the curve.

\section{Declarations}

\section{Ethics approval and consent to participate}

This study was approved by the Institutional Review Board of the Seoul National University Bundang Hospital.

\section{Consent for publication}

Not applicable.

\section{Availability of data and materials}


All relevant data are included in this manuscript. Additional data may be requested by contacting the corresponding author.

\section{Competing interests}

The authors declare that they have no competing interests.

\section{Funding}

This study was supported by the Seoul National University Bundang Hospital Research Fund. The funding source had no role in study design, data collection, analysis or interpretation, or in writing the manuscript. The article processing fee would be expensed using the funding received.

\section{Authors' contributions}

JSK and GSM equally contributed to the drafting of manuscript. JSK processed and analyzed the data, added in interpreting the results, worked on the manuscript. YJC designed figures and tables and worked out the technical details. HSG conceived and supervised the study with critical revision. All authors have read and approved the manuscript.

\section{Acknowledgments}

The authors thank Division of Statistics in Medical Research Collaborating Center at Seoul National University Bundang Hospital for statistical analyses.

\section{References}

1. Mondelli M, Giannini F, Ballerini M, Ginanneschi F, Martorelli E. Incidence of ulnar neuropathy at the elbow in the province of Siena (Italy). J Neurol Sci. 2005;234(1-2):5-10.

2. William F. The role of the cubital tunnel in tardy ulnar palsy. Can J Surg. 1957;2(5057):1344-5.

3. Bäumer P, Dombert T, Staub F, et al. Ulnar Neuropathy at the Elbow: MR Neurography-Nerve T2 Signal Increase and Caliber. Radiology. 2011;260(1):199-206.

4. Robertson C, Saratsiotis J. A Review of Compressive Ulnar Neuropathy at the Elbow. J Manipulative Physiol Ther. 2005;28(5):345.

5. Bartels RHMA, Verhagen WIM, van der Wilt GJ, Meulstee J, van Rossum LGM, Grotenhuis JA. Prospective Randomized Controlled Study Comparing Simple Decompression versus Anterior Subcutaneous Transposition for Idiopathic Neuropathy of the Ulnar Nerve at the Elbow: Part 1. Neurosurgery. 2005;56(3):522-30.

6. Charles YP, Coulet B, Rouzaud J-C, Daures J-P, Chammas M. Comparative Clinical Outcomes of Submuscular and Subcutaneous Transposition of the Ulnar Nerve for Cubital Tunnel Syndrome. $J$ Hand Surg. 2009;34(5):866-74. 
7. Dellon AL, Coert JH. Results of the Musculofascial Lengthening Technique for Submuscular Transposition of the Ulnar Nerve at the Elbow. J Bone Joint Surg Am. 2003;85(7):1314-20.

8. Foster RJ, Edshage S. Factors related to the outcome of surgically managed compressive ulnar neuropathy at the elbow level. J Hand Surg. 1981;6(2):181-92.

9. Zaidman CM, Seelig MJ, Baker JC, Mackinnon SE, Pestronk A. Detection of peripheral nerve pathology: Comparison of ultrasound and MRI. Neurology. 2013;80(18):1634-40.

10. Hold A, Mayr-Riedler MS, Rath T, et al. 3-Tesla MRl-assisted detection of compression points in ulnar neuropathy at the elbow in correlation with intraoperative findings. J Plast Reconstr Aesthet Surg. 2018;71(7):1004-9.

11. Vucic S, Cordato DJ, Yiannikas $C$, Schwartz RS, Shnier RC. Utility of magnetic resonance imaging in diagnosing ulnarneuropathy at the elbow. Clin Neurophysiol. 2006;117(3):590-5.

12. American Association of Electrodiagnostic Medicine. America Association of Physical medicine and Rehabilitation. Practice parameter for electrodiagnostic studies in ulnar neuropathy at the elbow: Summary statement. Muscle Nerve. 1999;22(3):408-11.

13. Goldberg G, Light BJ, Blair TR. SJ. Ulnar neuropathy at the elbow: Results of medial epicondylectomy. J Hand Surg. 1989;14(2):182-8.

14. Wilson $D H$, Krout $R$. Surgery of ulnar neuropathy at the elbow: 16 cases treated by decompression without transposition. J Neurosurg. 1973,38(6):780-5.

15. Chang K-V, Wu W-T, Han D-S, Özçakar L. Ulnar Nerve Cross-Sectional Area for the Diagnosis of Cubital Tunnel Syndrome: A Meta-Analysis of Ultrasonographic Measurements. Arch Phys Med Rehabil. 2018;99(4):743-57.

16. Podnar S, Omejec G, Bodor M. Nerve conduction velocity and cross-sectional area in ulnar neuropathy at the elbow: NCV and CSA. Muscle Nerve. 2017;56(6):E65-72.

17. Terayama Y, Uchiyama S, Ueda K, et al. Optimal Measurement Level and UInar Nerve Cross-Sectional Area Cutoff Threshold for Identifying Ulnar Neuropathy at the Elbow by MRI and Ultrasonography. J Hand Surg. 2018;43(6):529-36.

18. Fullerton PM, Gilliatt RW. Median and ulnar neuropathy in the guinea-pig. J Neurol Neurosurg Psychiatry. 1967;30(5):393-402.

19. Power HA, Sharma K, El-Haj M, Moore AM, Patterson MM, Mackinnon SE. Compound Muscle Action Potential Amplitude Predicts the Severity of Cubital Tunnel Syndrome. J Bone Joint Surg Am. 2019;101(8):730-8.

20. Gupta R, Truong L, Bear D, Chafik D, Modafferi E, Hung CT. Shear stress alters the expression of myelin-associated glycoprotein (MAG) and myelin basic protein (MBP) in Schwann cells. J Orthop Res. 2005;23(5):1232-9.

21. Tapadia M, Mozaffar T, Gupta R. Compressive Neuropathies of the Upper Extremity: Update on Pathophysiology, Classification, and Electrodiagnostic Findings. J Hand Surg. 2010;35(4):668-77. 
22. Beekman R, Wokke JHJ, Schoemaker MC, Lee ML, Visser LH. CME Ulnar neuropathy at the elbow. Neurology. 2004;63(9):1675-80.

23. La Torre D, Raffa G, Pino MA, et al. A Novel Diagnostic and Prognostic Tool for Simple Decompression of Ulnar Nerve in Cubital Tunnel Syndrome. World Neurosurg. 2018;118:e964-73.

24. Shi Q, MacDermid JC, Santaguida PL, Kyu HH. Predictors of Surgical Outcomes Following Anterior Transposition of Ulnar Nerve for Cubital Tunnel Syndrome: A Systematic Review. J Hand Surg. 2011;36(12):1996-2001..e6.

25. Matsuzaki H, Yoshizu T, Maki Y, Tsubokawa N, Yamamoto Y, Toishi S. Long-term clinical and neurologic recovery in the hand after surgery for severe cubital tunnel syndrome. $J$ Hand Surg. 2004;29(3):373-8.

\section{Figures}

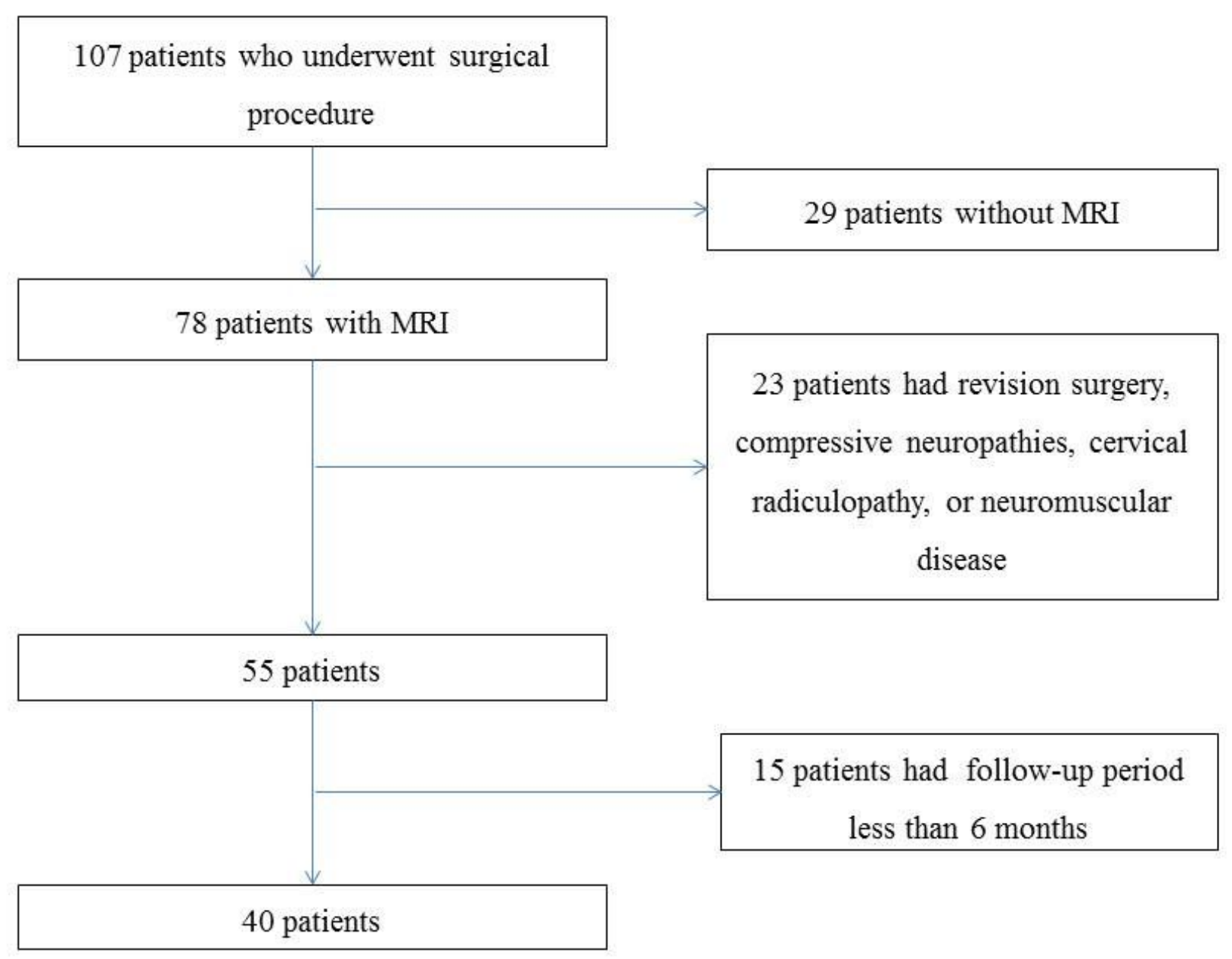

Figure 1 
This flowchart details the patients who were included in this study. EDX = Electrodiagnostic test.

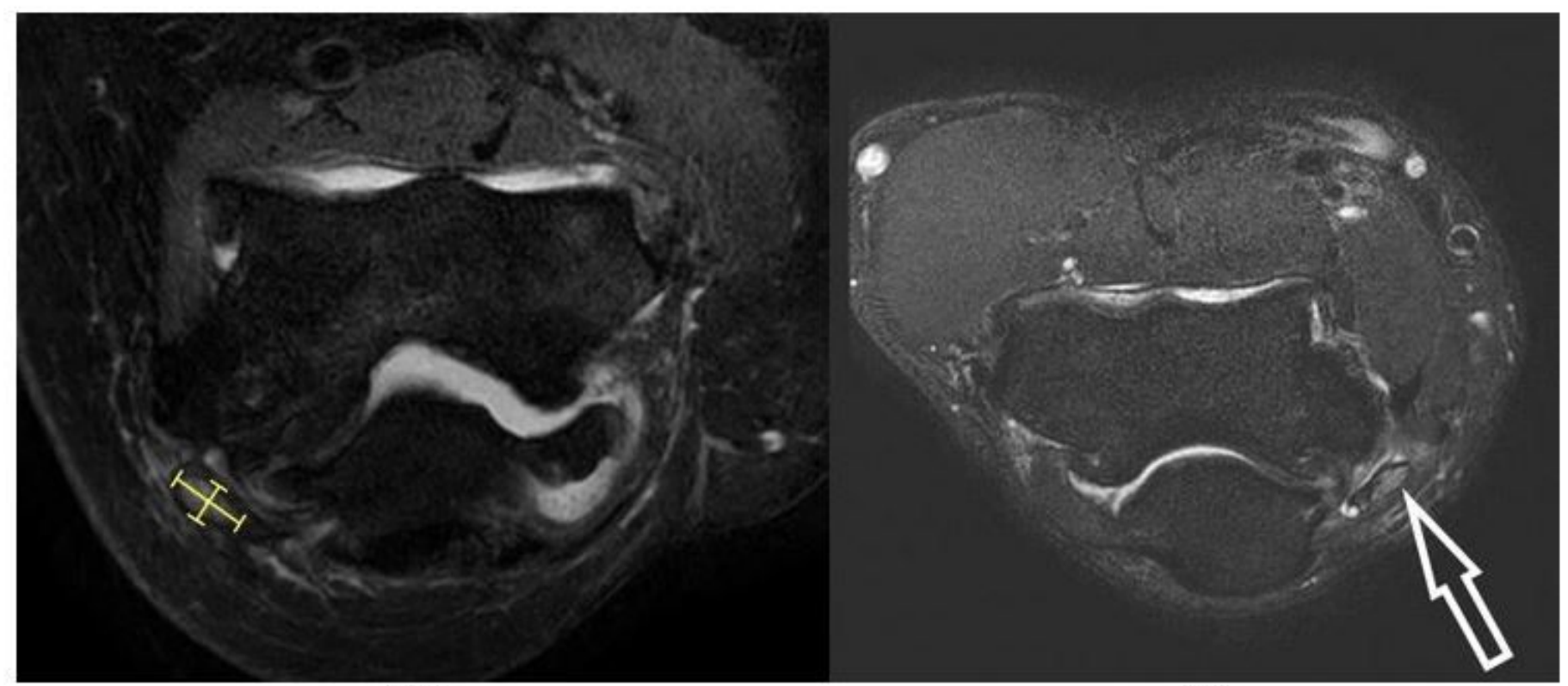

(A)

(B)

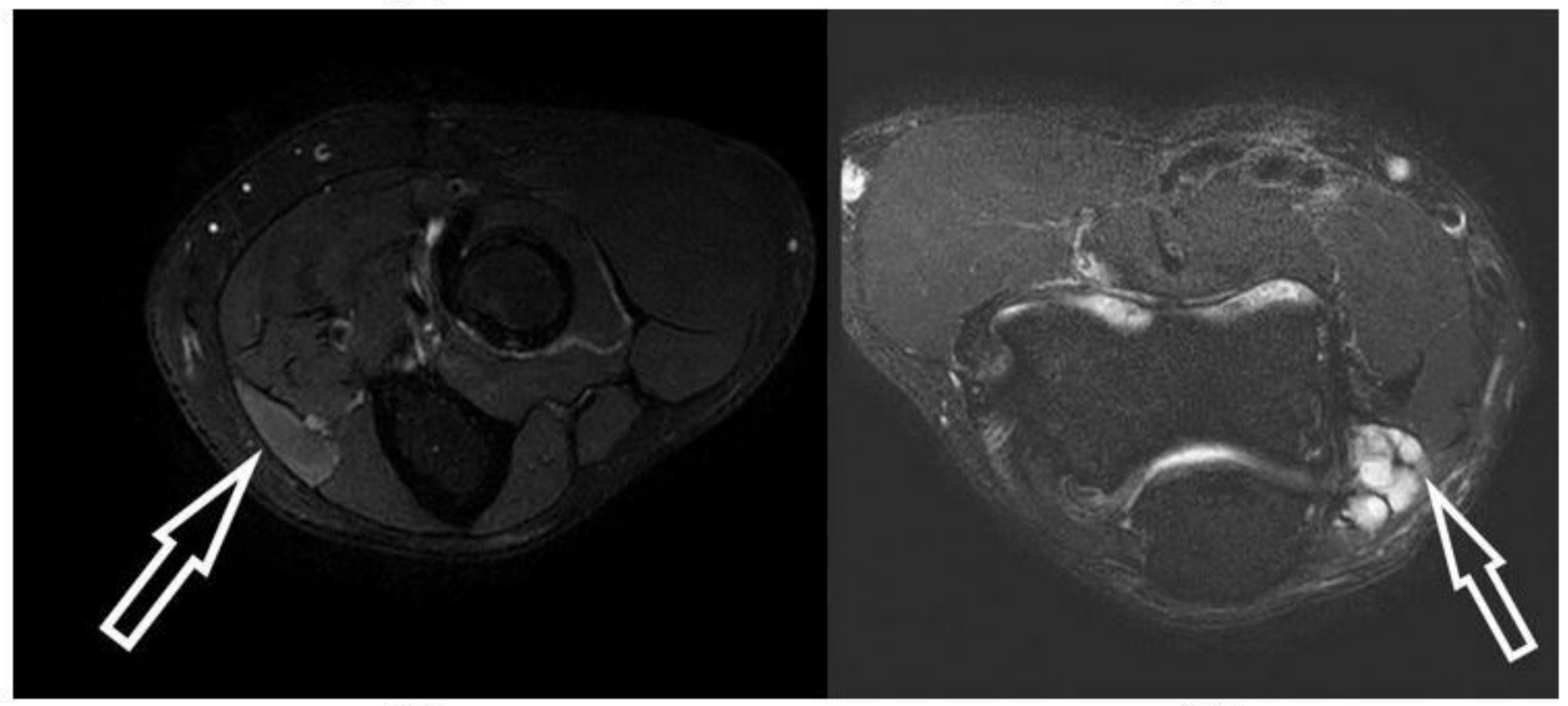

(C)

(D)

\section{Figure 2}

(A) This section of MRI shows the measurement of the ulnar nerve diameter for calculation of ulnar nerve cross sectional area in T1 axial sequence image of MRI. (Long diameter: $8.3 \mathrm{~mm}$, Short diameter: $3.7 \mathrm{~mm}$ ) (B) This section of MRI indicates the nerve signal intensity change marked by white arrow in T2 axial sequence image of MRI. (C) This section of MRI indicates the muscle signal intensity change of FCU marked by white arrow in T2 axial sequence image of MRI. FCU = Flexor carpi ulnaris muscle. (D) The presence of ganglion causing compression of the ulnar nerve was detected at cubital tunnel (marked by white arrow) in T2 axial sequence image of MRI. 


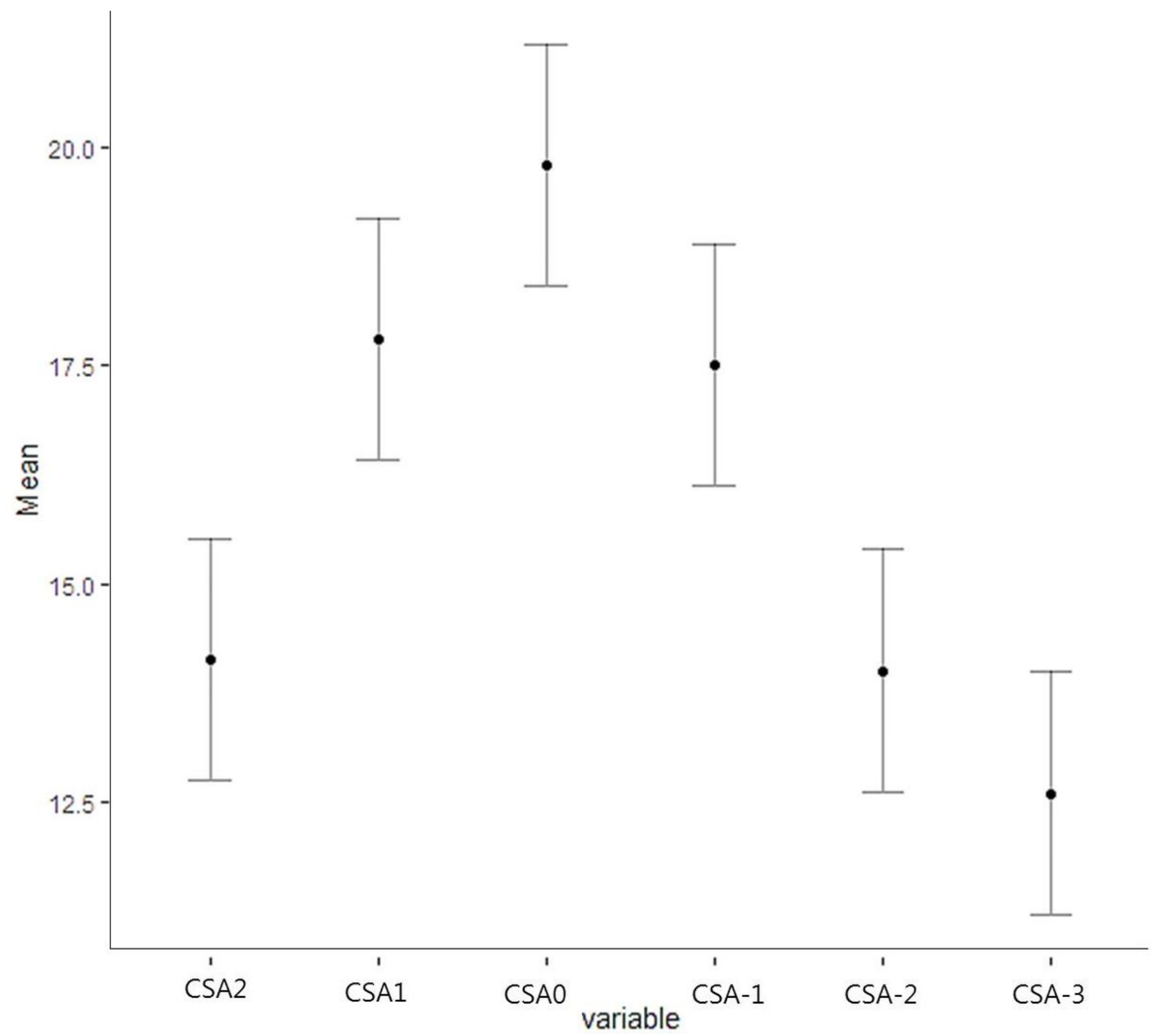

Figure 3

This chart shows the distribution of ulnar nerve CSA. CSA = cross-sectional area; CSA2 = CSA at $2 \mathrm{~cm}$ proximal to the medial epicondyle; $C S A O=C S A$ at the medial epicondyle; $C S A-3=C S A$ at $3 \mathrm{~cm}$ distal to the medial epicondyle. 


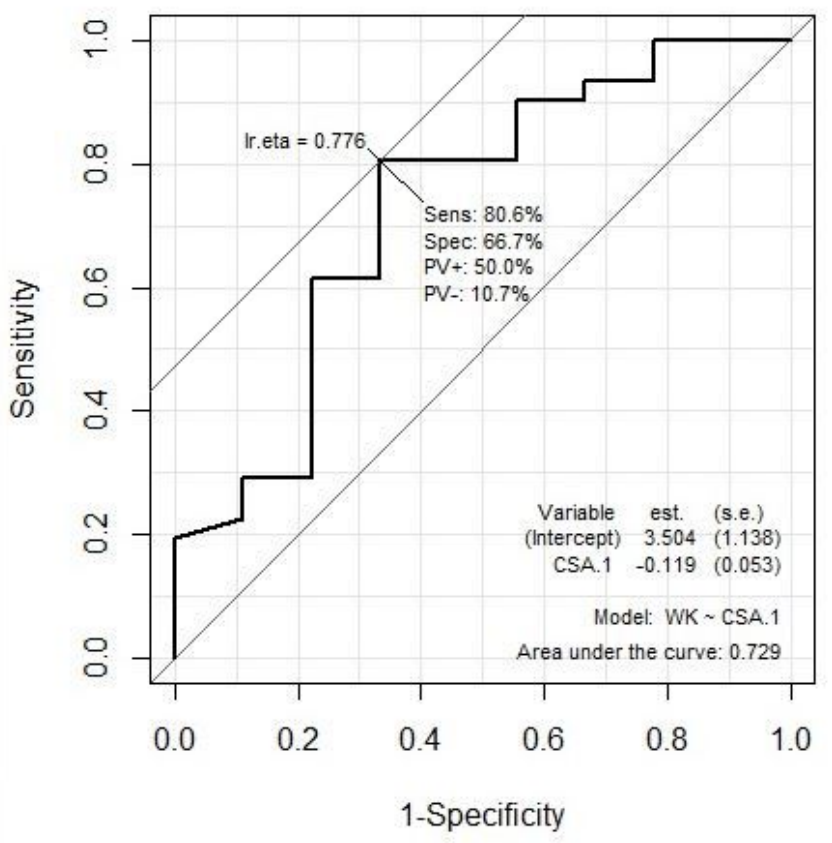

(A)

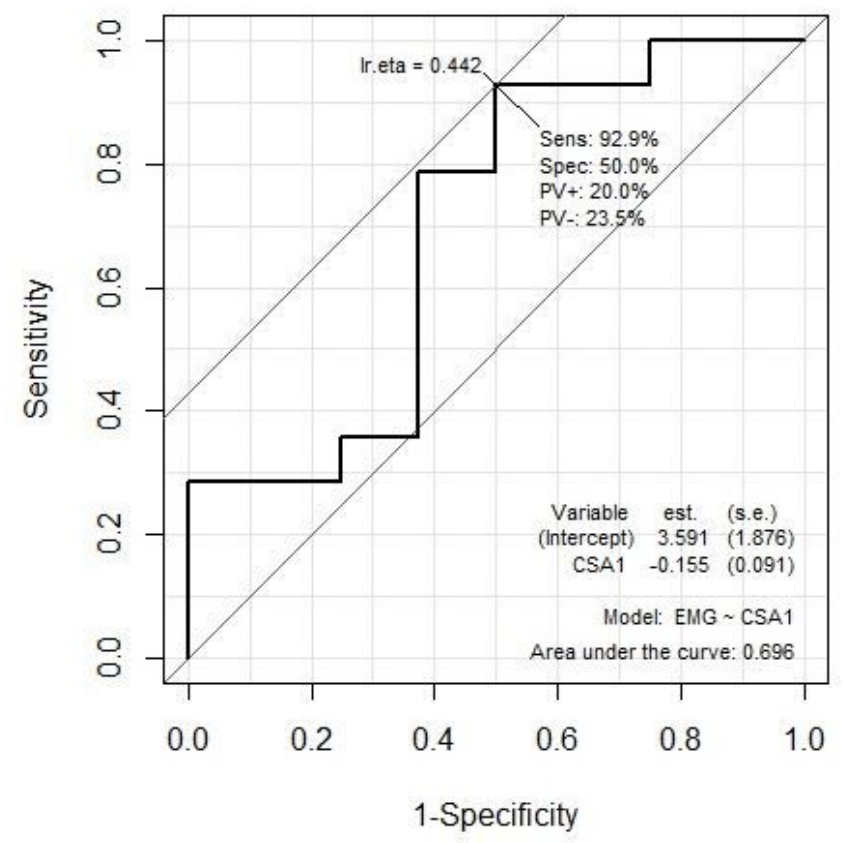

(B)

\section{Figure 4}

This graph shows the cutoff value and receiver operating characteristic curve. (A) The cut-off value of CSA at distal $1 \mathrm{~cm}$ was $18.9 \mathrm{~mm} 2$. Sensitivity and specificity at distal $1 \mathrm{~cm}$ were $80.6 \%$ and $66.7 \%$. The AUC for CSA at distal $1 \mathrm{~cm}$ was 0.729. (B) The cut-off value of CSA at proximal $1 \mathrm{~cm}$ was $16.7 \mathrm{~mm} 2$.

Sensitivity and specificity at proximal $1 \mathrm{~cm}$ were $92.9 \%$ and $50.0 \%$. The AUC for CSA at proximal $1 \mathrm{~cm}$ was 0.724 .

\section{Supplementary Files}

This is a list of supplementary files associated with this preprint. Click to download.

- Additionalfile1.docx 